\title{
Evaluation of the efficacy in visual function improvement of brolucizumab with aflibercept in neovascular age-related macular degeneration
}

Magdalena Musiał-Kopiejka ${ }^{1}$, Katarzyna Polanowska' ${ }^{1}$, Dariusz Dobrowolski², Beniamin Oskar Grabarek ${ }^{3}$, Anita Lyssek-Boroń ${ }^{1,4 *}$

'Department of Ophthalmology with Paediatric Unit, ${ }^{\text {th }}$ Regional Hospital, Medykow Square 1, Sosnowiec, Poland; ${ }^{2}$ Chair and Clinical Department of Ophthalmology, Faculty of Medical Sciences in Zabrze, Medical University of Silesia, Katowice, Poland;
${ }^{3}$ Department of Histology, Cytophysiology and Embryology, Faculty of Medicine in Zabrze, University of Technology in Katowice, Zabrze, Poland $;{ }^{4}$ Department of Ophtamology, Faculty of Medicine in Zabrze, University of Technology in Katowice, Poland

\section{Background;}

Age-related macular degeneration (AMD) is the leading cause of blindness among the elderly in the developed world. Currently, there are 4 available intravitreal anti-VEGF therapies (pagaptanib, bevacizumab ranimizumab and aflibercept). Brolucizumab is a newly developed anti-VEGF molecule for neowascular AMD treatment. Based primarily on the results of the phase III HAWK and HARRIER trias brolucizumab was recently approved in the US for the treatment of exudative (wet) AMD. In this paper we summarize the safety, visual and anatomic outcomes of brolucizumab in the treatment of neovascular AMD.

Aim:

The aim of the study was to evaluate the efficacy in visual function improvement of brolucizumab with aflibercept in neovascular age-related macular degeneration (AMD).

\section{Material and methods:}

Fourteen patients, aged $>50$ years, with untreated, active choroidal neovascularization due to age-related macular degeneration in the study eye. Eligible participants were randomized 1:1 to intravitreal injections of brolucizumab $(6 \mathrm{mg} / 50 \mu \mathrm{l})$ or aflibercept $(2 \mathrm{mg} / 50 \mu \mathrm{l})$. Both groups received 3 monthly loading doses and were then treated every 8 weeks. Brolucizumab demonstrate non inferiority to aflibercept in BCVA change from baseline.

\section{Results:}

Optical Coherence Tomography Angiography( angio-OCT, OCTA) is the latest diagnostic tool In ophthalmology. It allows to visualize blood flow in the vessels of the retina and choroid In a non invasive manner. Angio-OCT is based on technology that uses laser ligt reflection from the surface of moving red blood cells. The movement of erythrocytes enables the visualization of the flow In the blood vessels. OCTA algorithms create an images( from $3 \mathrm{~mm} 2$ to $12 \mathrm{~mm} 2$ ) that are normalny divided into four zones: superficial retinal lexus, deep retinal lexus, outer retina and choriocapillaries
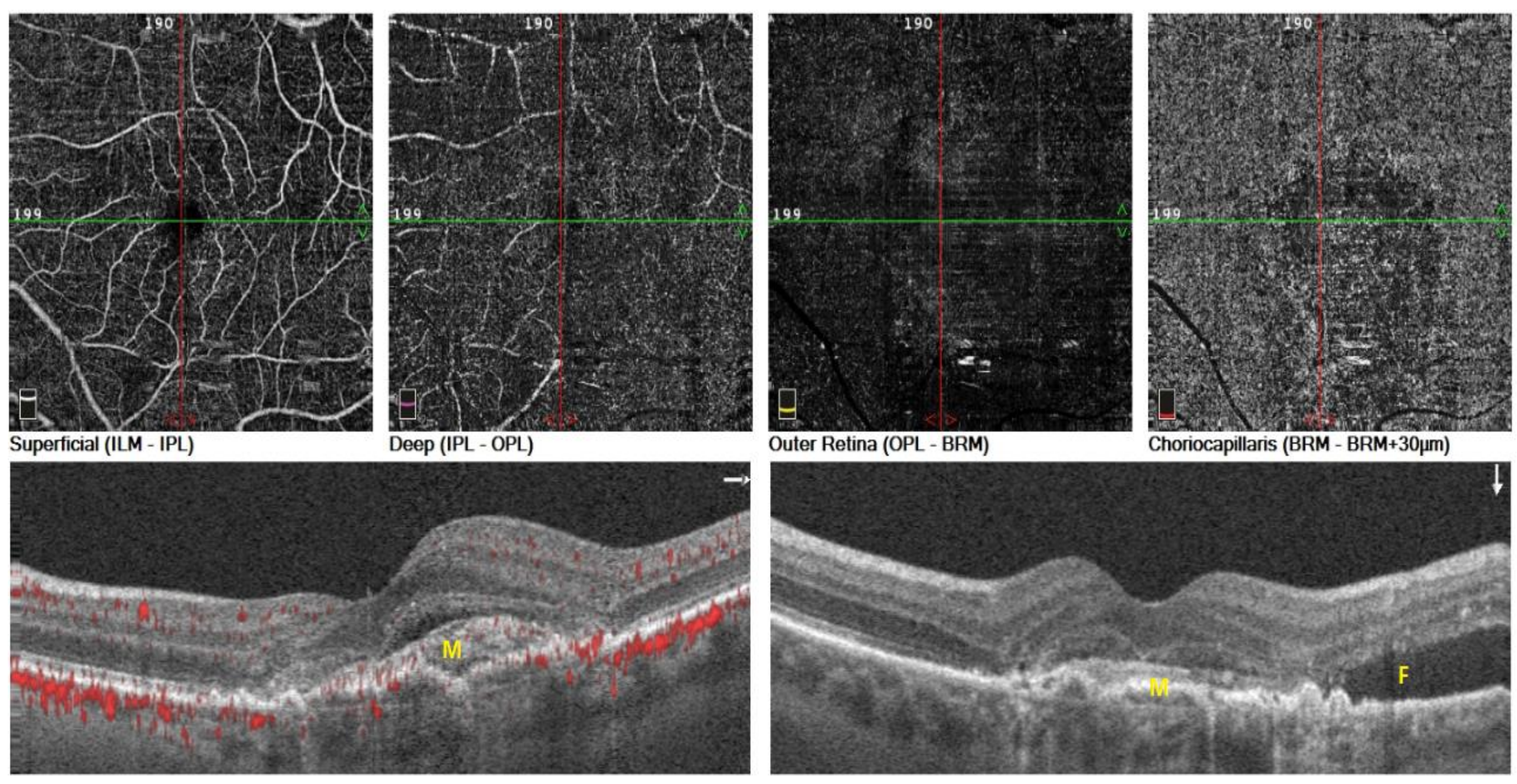

lar degeneration. OCTA shows a neovascular membrane (M) with subretinal fluid (F) before first brolacizumab intravitreal injection.
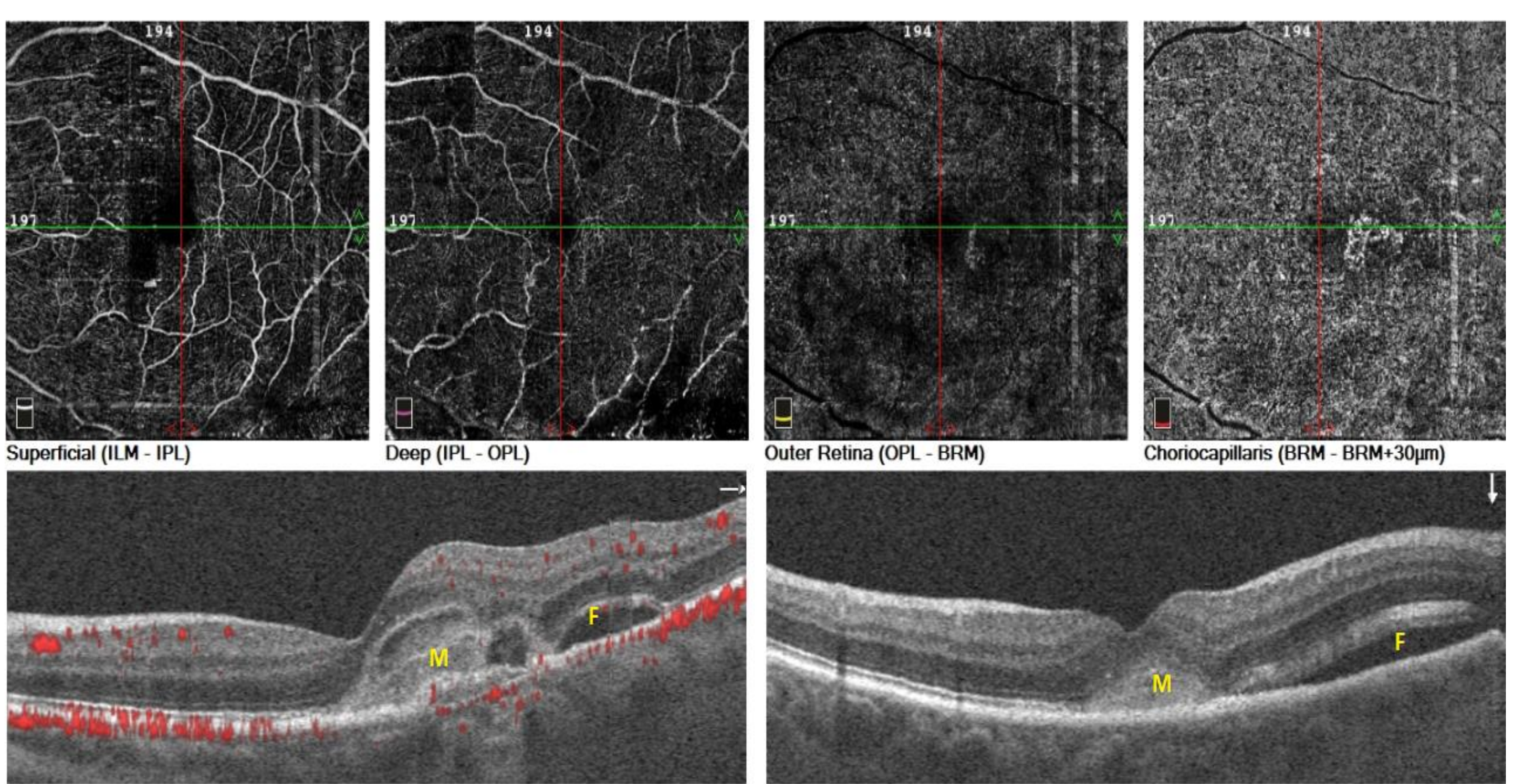

An angioOCT of 74-year-old man with neovascular age-related macular degeneration. OCTA shows a neovascular membrane (M)with subretinal fluid $(\mathbf{F})$ before first brolacizumab intravitreal injection
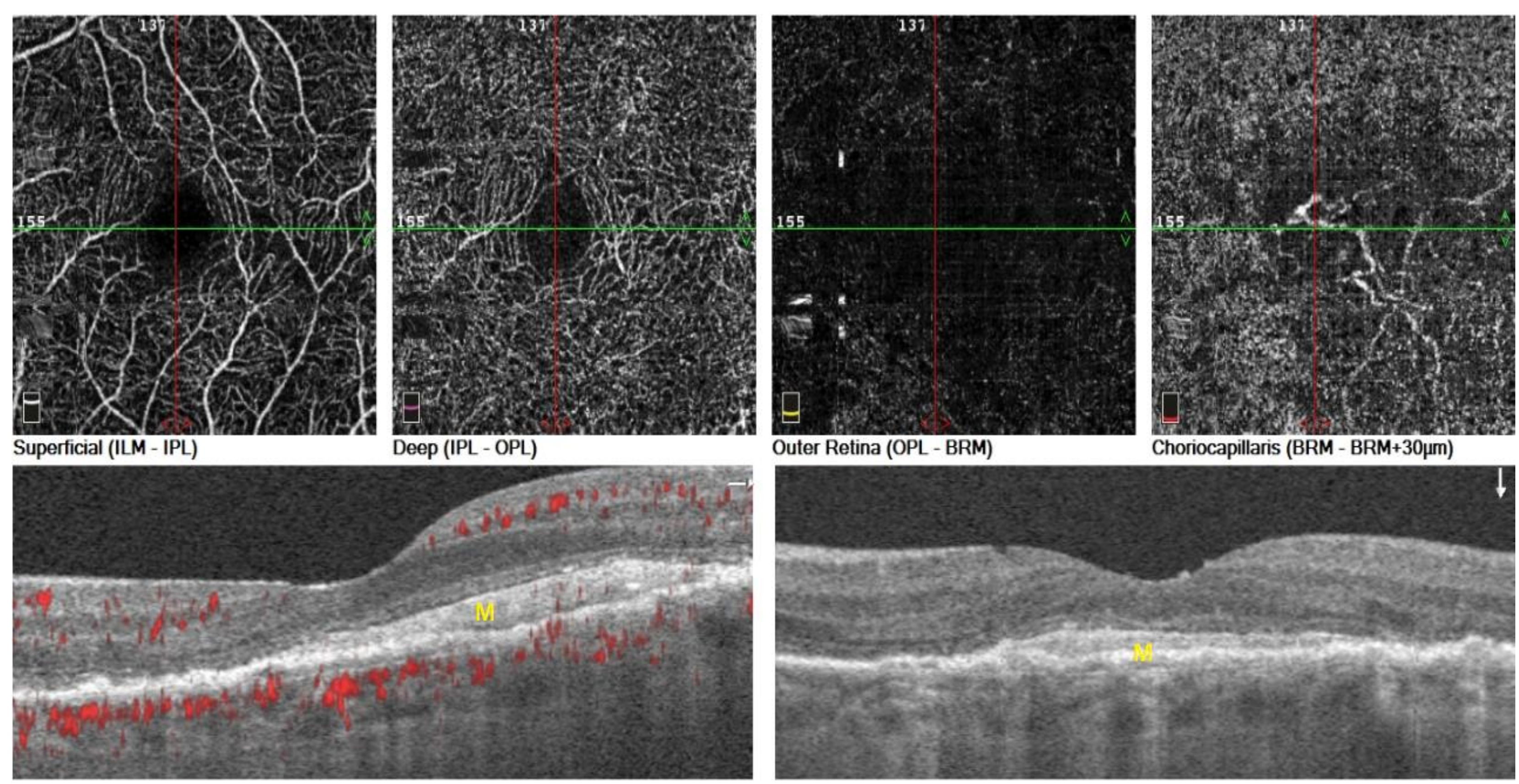

AngioOCT after monthly brolacizumab intravitreal injections, with complete resolution of subretinal fluid( $\mathrm{F}$ ). Improvement in Pelli Robson contrast sensitivity chart tests and decrease of matamorphopsia In Amsler test were observed
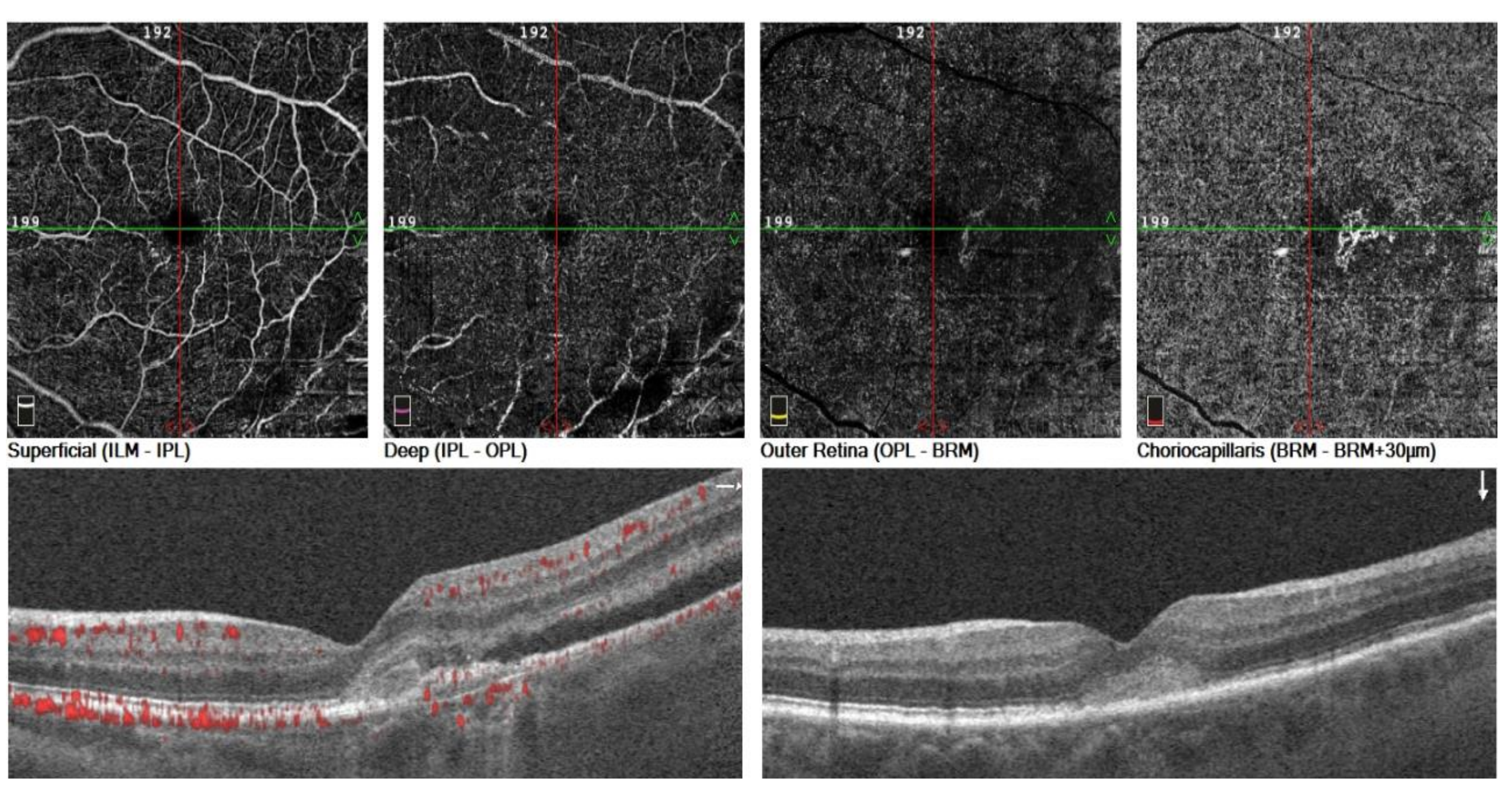

AngioOCT after monthly brolacizumab intravitreal injections

\section{Conclussion:}

Brolucizumab was superior to aflibercept in visual function like contrast sensitivity and decrease of metamorphopsia. Anatomic outcomes favored brolucizumab over aflibercept. 